M. KOPISZ (Wrockaw)

\title{
COUPLED FIELDS IN A PIEZOELECTRIC BODY
}

1. Introduction. Piezoelectricity in a material deformable continuum represents a conjugation of mechanical fields (deformations, stresses of a body) with an electric field. Under the effect of mechanical stress in a piezoelectric body an electric polarization appears, and conversely, an external electric field applied results in a deformation of the piezoelectric body.

Piezoelectric effects have found a large practical application but lack a complete theoretical description. The theory of piezoelectricity elaborated by W. Voight [8] has some drawbacks. According to this theory the piezoelectric effects cannot occur in an isotropic body or in crystals with central symmetry. Nevertheless experimental investigations show that the piezoelectric effect can occur in centrosymmetric crystals. Some other anomalies occur which are not anticipated by the theory and which are disclosed by the experiments of Mead [4]. Some of them are explained by the gradient theory of R. Mindlin [5].

In the present paper we give a description of coupled fields in a piezoelectric based on a general method of conjugation of mechanical and electromagnetic fields in a deformable body which are generated by some differential operators, as was shown in [2], [3]. The essential new assumptions of the presented method are a generalized formula for the Lorentz force (which may be non-zero in the absence of free currents and electric charges in the body) and the omission of the symmetry condition for the deformation and stress tensors, the condition that may be violated in the case of mutual action of an electromagnetic field and a material deformable continuum.

2. A deformable body in an electric field. Now, using the results of [2], [3] where a general method of conjugation of mechanical and electromagnetic fields generated by differential operators is presented we discuss the 
case of a deformable continuum in a constant electric field. By the Maxwell equations the electric and magnetic phenomena can only be considered separately in the electrostatic $(\partial E / \partial t=0)$ or magnetostatic $(\partial B / \partial t=0)$ case. Here piezoelectricity is understood as a non-trivial conjugation of mechanical and electrostatic fields in a material deformable body.

The antisymmetric stress tensor $\mathcal{F}^{\alpha \beta}(\mathbf{D}, \mathbf{H})$ (of an electromagnetic field introduced in [2] has now the simplified form

$$
\mathcal{F}^{\alpha \beta}=\left[\begin{array}{cccc}
0 & -D^{1} & -D^{2} & -D^{3} \\
D^{1} & & & \\
D^{2} & 0 & \\
D^{3} & & &
\end{array}\right],
$$

where $D^{i}$ denotes the electric induction vector, whereas the magnetic induction vector $H^{i}=0$.

The antisymmetric deformation tensor $\mathcal{G}_{\alpha \beta}(\mathbf{E}, \mathbf{B})$ of an electromagnetic field reduces to

$$
\mathcal{G}_{\alpha \beta}=\left[\begin{array}{cccc}
0 & E_{1} & E_{2} & E_{3} \\
-E_{1} & & & \\
-E_{2} & & 0 & \\
-E_{3} & & &
\end{array}\right],
$$

where $E_{i}$ denotes the electric field vector, whereas the magnetic field vector $B_{i}=0$.

Let us remark that throughout the paper the Greek indices of tensor functions vary in $\{0,1,2,3\}$, whereas the Latin ones in $\{1,2,3\}$.

In [2], it was shown that the space-time equilibrium equations for a deformable medium in an electromagnetic field have the form

$$
\nabla_{\mu} F_{. \nu}^{\mu}=F_{\nu}+{ }_{\mathrm{e}} F_{\nu}
$$

where ${ }_{\mathrm{e}} F_{\nu}$ denotes the Lorentz force, $F_{\nu}$ the external mass force acting on the body and $F^{\mu}{ }_{\nu}$ the stress four-tensor of the material continuum.

Equations (3a) should be completed by the Maxwell equations for the tensors $\mathcal{F}^{\alpha \beta}$ and $\mathcal{G}_{\alpha \beta}$ as follows:

$$
\begin{aligned}
& \nabla_{\alpha} \mathcal{F}^{\alpha \beta}=I^{\beta}, \\
& \nabla_{\alpha} \widehat{\mathcal{G}}^{\alpha \beta}=0,
\end{aligned}
$$

where $\widehat{\mathcal{G}}^{\alpha \beta}$ is the dual tensor to $\mathcal{G}_{\alpha \beta}$ and $I^{\beta}$ denotes the four-tensor of current.

Let us indicate that equation (3c) becomes an identity if

$$
\mathcal{G}_{\alpha \beta}=\nabla_{[\alpha} A_{\beta]},
$$

where [] denotes the antisymmetric part of a tensor. 
Conjugation of equations (3a) and (3b) is done by means of the formula for the Lorentz force ${ }_{e} F_{\nu}$. It is the force of an electromagnetic field affecting the matter, especially currents and charges. By tradition [1] the formula takes the form

$$
\mathrm{e}^{F^{\beta}}=\mathcal{G}^{\beta \nu} I_{\nu} .
$$

The Lorentz force (5a) and the resulting form of conjugation was assumed in [2]. In [3] a more general form of the formula for the Lorentz force was adopted and some arguments for it were presented. Similarly, in the present paper we take

$$
{ }_{\mathrm{e}} F^{\beta}=\mathcal{G}^{\beta \nu} I_{\nu}+\frac{1}{4} \mathcal{F}_{\mu \lambda} \nabla^{\beta} \mathcal{G}^{\mu \lambda}-\frac{1}{4} \mathcal{G}^{\mu \lambda} \nabla^{\beta} \mathcal{F}_{\mu \lambda} .
$$

Formula (5b) shows that some material media may exist for which the constitutive relations (polarization laws) for the tensors $\mathcal{F}^{\alpha \beta}, G_{\alpha \beta}$ are such that (5b) becomes (5a). Also, let us remark that two new terms of (5b) describe the force with which the electromagnetic field acts on the matter without free charges and currents but with the possibility of polarization. Here we consider precisely that medium $\left(I^{\nu}=0\right)$ in the electrostatic field.

Taking the traditional form (5a) of the formula for the Lorentz force as for example in [5], [6] we conclude that the equilibrium equations (3a) are not conjugated with equations $(3 \mathrm{~b})$ describing the electromagnetic field, since ${ }_{\mathrm{e}} F^{\beta}=0$ when no external charges and currents occur. Therefore, in the traditional approach the electric and magnetic effects are conjugated in the case of piezoelectric only in the constitutive relations.

In the classical Voight theory [8] we take the algebraic linear material relations of the form

$$
\begin{aligned}
T_{i j} & =c_{i j k l} \varepsilon_{k l}-\eta_{k i j} E_{k}, \\
D_{i} & =\eta_{i k l} \varepsilon_{k l}+\varkappa_{i k} E_{k} .
\end{aligned}
$$

In the above formulas $T_{i j}, \varepsilon_{i j}$ denote the three-tensors of stresses and deformations of the body, respectively; $\eta_{i k l}$ are the piezoelectric constants, $\varkappa_{i k}$ the dielectric constants, and $c_{i j k l}$ the elasticity constants.

In the case of an isotropic body the tensors of material constants should be of the form

$$
\begin{aligned}
c_{i j k l} & =\mu\left(\delta_{i k} \delta_{j l}+\delta_{i l} \delta_{j k}\right)+\lambda \delta_{i j} \delta_{k l}, \\
\eta_{k i j} & =e e_{k i j}, \\
\varkappa_{i j} & =\varkappa \delta_{i j},
\end{aligned}
$$

where $e_{k i j}$ is the antisymmetric Ricci tensor.

Consequently, based on (6), (7) we conclude that there is no piezoelectric effect in an isotropic body because the term $e \dot{e}_{k i j} E_{k}$ must vanish as a result of the symmetry of the stress tensor $T_{i j}$ postulated in the classical 
theory. This conclusion of the symmetric Voight theory of piezoelectricity is contradicted by experiment [4].

The use of the symmetric stress tensor $T_{i j}$, and consequently of the symmetric deformation tensor $\varepsilon_{i j}$, is traditionally based on conservation laws, of angular momentum in particular.

In [3] the principle of conservation of momentum, angular momentum and energy for a deformable medium in an electromagnetic field was discussed and it was proved that the validity of those principles for a system composed of an electromagnetic field and a deformable body implies only the condition

$$
T_{[i j]}+{ }_{\mathrm{e}} T_{[i j]}^{(\mathrm{M})}=0,
$$

where $T_{i j}$ denotes the so-called Maxwell stress tensor of the electromagnetic field.

In [3] the four-tensor of momentum-energy ${ }_{\mathrm{e}} T_{\alpha}^{\beta}$ of an electromagnetic field is introduced by

$$
\mathrm{e}^{T_{\alpha}^{\beta}}=\mathcal{F}_{\alpha \nu} \mathcal{G}^{\nu \beta}+\frac{1}{4} \delta_{\alpha}{ }^{\beta} \mathcal{F}_{\mu \nu} \mathcal{G}^{\mu \nu}
$$

This tensor is related to the generalized Lorentz force ${ }_{\mathrm{e}} F^{\beta}$ in the following way:

$$
\nabla_{\alpha \mathrm{e}} T^{\alpha \beta}=-{ }_{\mathrm{e}} F^{\beta}
$$

The space components of the momentum-energy tensor ${ }_{\mathrm{e}} T_{\alpha}^{\beta}$ determine the Maxwell stress tensor ${ }_{\mathrm{e}} T_{i j}^{(\mathrm{M})}$ with

$$
\mathrm{e}^{(\mathrm{M})}=-{ }_{\mathrm{e}} T_{i j} \text {. }
$$

Let us remark that the validity of condition (8) does not imply necessarily that the antisymmetric parts of each tensor appearing in that formula vanish. As was pointed out in [3] the Maxwell stress tensor ${ }_{\mathrm{e}} T_{i j}^{(\mathrm{M})}$ of an electromagnetic field is not symmetric in general, and as a consequence the stress tensor $T_{i j}$ of a deformable body need not be symmetric either, in order that the principle of conservation given by ( 8 ) be satisfied.

In the present paper we give a simplest example of piezoelectric with algebraic material relations, and those relations together with the generalized Lorentz force formula result in the conjugation of equations (3a), (3b) and the appearance of a non-vanishing non-symmetric part of the stress tensors ${ }_{\mathrm{e}} T_{i j}^{(\mathrm{M})}, T_{i j}$.

We derive the displacement equations for such piezoelectric. Those equations constitute a generalization of the classical theory of Voight yielding the existence of an isotropic piezoelectric but they are different from those of the gradient theory of Mindlin [5] where different constitutive relations are introduced. 
Now, we consider the simplest case of an isotropic piezoelectric. Without the traditional restriction on the stress and deformation tensors $T_{i j}$ and $\varepsilon_{i j}$ to be symmetric we assume the following constitutive relations for an isotropic piezoelectric:

$$
\begin{gathered}
T_{i j}=\mu \varepsilon_{i j}+\alpha \varepsilon_{j i}+\lambda \delta_{i j} \varepsilon_{k k}-e e_{i m n} \varepsilon_{m n} E_{j}, \\
D_{i}=e e_{i k j} \varepsilon_{k j}+\varkappa E_{i} .
\end{gathered}
$$

Let us remark that formulae (10a), (10b) as well as the constitutive relations (6), (7) of Voight theory preclude the piezoelectric effect in an isotropic body if the symmetry of stresses and deformations is required.

Further, we show that formulae (10a), (10b) imply the validity of the angular momentum conservation principle in the sense of condition (8) for the antisymmetric parts of the stress tensors of the material body and the electromagnetic field. We give the explicit form of ${ }_{\mathrm{e}} T_{i j}^{(\mathrm{M})}, T_{i j}$.

A generalization of (10a), (10b) to non-isotropic bodies leading to the validity of (8) for the angular momentum is

$$
\begin{gathered}
T_{i j}=c_{(i j) k l} \varepsilon_{k l}+\eta_{k(i j)} E_{k}-\beta_{i m n} \varepsilon_{m n} E_{j}-\varkappa_{i k} E_{i} E_{j}, \\
D_{i}=\beta_{i m n} \varepsilon_{m n}+\varkappa_{i k} E_{k},
\end{gathered}
$$

where () denotes the symmetric part of a tensor.

The above formulae constitute a generalization of the constitutive relations (6a), (6b) of Voight theory. Let us also remark that (10a) can be completed by a completely symmetric term $\varkappa E_{i} E_{j}$ for an isotropic piezoelectric without violation of condition (8).

3. Displacement equilibrium equations of an isotropic piezoelectric. Here we consider an isotropic piezoelectric on which no external charges or current are grouped, that is, $I_{\mu} \equiv 0$. As was shown in [2] the motion equations of a deformable continuum in an electromagnetic field have the form

$$
\nabla_{j} T_{. i}^{j}+{ }_{\mathrm{e}} F_{i}+F_{i}=D P_{i},
$$

where ${ }_{\mathrm{e}} F_{i}$ denotes the generalized Lorentz three-force and where formula (5b) for the four-vector ${ }_{\mathrm{e}} F_{\mu}$ is postulated. $D(\ldots)$ is the so-called material derivative with respect to time.

Hence, after some easy transformations using the form of the tensors $\mathcal{F}^{\alpha \beta}, \mathcal{G}_{\alpha \beta}$ describing the electromagnetic field [3] we obtain a general formula for the space component of the Lorentz force $F_{i}$ :

$$
\begin{aligned}
\mathrm{e} F_{i}= & -\rho E_{i}-e_{i k p} B^{p} I^{k}+\frac{1}{2}\left(E_{k} \nabla_{i} D^{k}-D^{k} \nabla_{i} E_{k}\right) \\
& +\frac{1}{2}\left(H_{k} \nabla_{i} B^{k}-B^{k} \nabla_{i} H_{k}\right) .
\end{aligned}
$$


In the considered case of no external sources we have

$$
{ }_{\mathrm{e}} F_{i}=\frac{1}{2}\left(E_{k} \nabla_{i} D^{k}-D^{k} \nabla_{i} E_{k}\right) .
$$

Substituting (14) into (12) results in equations of motion for a deformable continuum in an electric field of intensity $E_{k}$ and electric induction $D^{k}$. Taking into account the constitutive relations (11a), (11b) we obtain the description of motion for a piezoelectric in terms of deformations. Assuming the constitutive relations $(10 \mathrm{a}),(10 \mathrm{~b})$ we obtain the deformation equations of motion for an isotropic piezoelectric. In that case the Lorentz force ${ }_{\mathrm{e}} F_{i}$ can be written as follows:

$$
{ }_{\mathrm{e}} F_{i}=\frac{1}{2} e e_{k m n}\left(E_{k} \nabla_{i} \varepsilon_{m n}-\varepsilon_{m n} \nabla_{i} E_{k}\right) .
$$

The deformation tensor $\varepsilon_{m n}$ appearing in (15) should be defined separately, assuming in that way a certain measure of deformation. Neither a specific form nor the symmetry of $\varepsilon_{m n}$ will be assumed.

Equations (12) should be completed by the conjugated Maxwell equations $(3 \mathrm{~b})$ for an electromagnetic field described by the stress tensor $\mathcal{F}^{\mu \nu}$, which leads, in the case of an electrostatic field, to the condition

$$
\nabla_{i} D^{i}=0 .
$$

The no sources Maxwell equations (3c) for the deformation tensor $\mathcal{G}_{\alpha \beta}$ are identically satisfied if (4) holds, with

$$
A_{\beta}=\left(\varphi, A_{i}\right),
$$

which, in the case of an electric field and the simplified form (2) of the tensor $\mathcal{G}_{\alpha \beta}$, is equivalent to the representation of the electric field $E_{i}$ by the gradient of a potential:

$$
E_{i}=\nabla_{i} \varphi .
$$

Equations (12), (15), (16), (17) and the material relations (10a), (10b) represent the equations of an isotropic piezoelectric. Let us underline right now that in the framework of the presented method we obtain equations more general than in the classical Voight theory [8] and different from those of [5], [6].

The deformation form of the equations for an isotropic piezoelectric in an electrostatic field described by a potential $\varphi$ is

$$
\begin{gathered}
\mu \nabla_{i} \varepsilon_{i j}+\alpha \nabla_{i} \varepsilon_{j i}+\lambda \delta_{i j} \nabla_{i} \varepsilon_{k k}-e e_{i m n} \varepsilon_{m n} \nabla_{j i} \varphi \\
+\frac{1}{2} e e_{k m n}\left(\nabla_{k} \varphi \nabla_{j} \varepsilon_{m n}-\varepsilon_{m n} \nabla_{j k} \varphi\right)+F_{j}=D P_{j}, \\
e e_{i m n} \nabla_{i} \varepsilon_{m n}+\varkappa \nabla_{i i} \varphi=0 .
\end{gathered}
$$

In the above equations five mass constants appear: the elasticity constants $\mu, \alpha, \lambda$, the dielectric constant $\varkappa$ and the piezoelectric constant $e$. 
The unknowns are the piezoelectric deformations $\varepsilon_{i j}$ and the electric potential $\varphi$ in the piezoelectric. Now, equations (18a), (18b) may result in different forms of displacement equations in dependence on the adopted deformation measure of a deformable body.

Let us remark that adopting the constitutive selations (10a) we delete the symmetry condition for the stress tensor $T_{i j}$ of the piezoelectric. It is natural, in that case, to adopt as measure of deformation of the body the following quantity obtained from the displacement vector $u_{j}$ :

$$
\varepsilon_{i j}=\nabla_{i} u_{j},
$$

also without the symmetry condition for that tensor. As a result, the generalized Lorentz force ${ }_{e} F_{i}$ does not vanish also for an isotropic piezoelectric without external charges and currents as formula (15) indicates. Consequently, the non-symmetric part of the Maxwell tensor $T_{i j}^{(\mathrm{M})}$ does not vanish according to (9a)-(9c). The antisymmetric parts of the stress tensor $T_{i j}$ of the piezoelectric and of the Maxwell stress tensor ${ }_{\mathrm{e}} T_{i j}^{(\mathrm{M})}$ satisfy identically condition (8), which is the conservation principle for the angular momentum; one can easily notice that

$$
\begin{aligned}
\mathrm{e}^{T_{i j}^{(\mathrm{M})}} & =D_{[i} E_{j]}=e \varepsilon_{m n} e_{m n[i} E_{j]}, \\
T_{[i j]} & =-e \varepsilon_{m n} e_{m n[i} E_{j]}=-{ }_{e} T_{i j}^{(\mathrm{M})} .
\end{aligned}
$$

Assuming the deformation measure of a body according to (19) equations (18a), (18b) now take the form

$$
\begin{gathered}
(2 \mu+\lambda) \cdot \operatorname{grad}_{i} \operatorname{div} \mathbf{u}+2 \alpha \nabla^{2} u_{j}+\frac{1}{2} e \nabla_{k} \varphi \nabla_{j}(\operatorname{rot} \mathbf{u})_{k} \\
-\frac{3}{2} e \nabla_{j k} \varphi(\operatorname{rot} \mathbf{u})_{k}+F_{j}=D P_{j}, \\
\varkappa \nabla^{2} \varphi=0 \Leftrightarrow \nabla^{2} \varphi=0 .
\end{gathered}
$$

The first term of equation (18b) vanishes because $\operatorname{div} \operatorname{rot}(.)=$.0 . Thus we have obtained four second order differential equations for the determination of the displacements $u_{i}$ of a piezoelectric and of the electric potential $\varphi$ in the piezoelectric.

Let us remark that besides some elasticity constants only the piezoelectric constant $e$ occurs in equations (22a), (22b). The dielectric constant $\varkappa$ is used for the determination of the electric induction $D^{k}$ from the material relation $(10 \mathrm{~b})$. Thus, it is used for the determination of the polarization vector $P_{k}$ of the body, which is usually defined as follows:

$$
D_{k}=\varkappa_{0} E_{k}+P_{k},
$$

where $\varkappa_{0}$ is the vacuum dielectric constant. 
Assuming the constitutive relation (10b) for an isotropic piezoelectric we express the polarization vector $P_{k}$ as follows:

$$
P_{k}=e(\operatorname{rot} \mathbf{u})_{k}+\left(\varkappa-\varkappa_{0}\right) E_{k} .
$$

Let us notice that in Mindlin's paper [5] the polarization vector $P_{k}$ is an unknown function introduced in the theory independently of the antisymmetric part of the deformation tensor $\varepsilon_{i j}$ because there the traditional symmetry condition is assumed.

In the present paper the polarization $P_{k}$ is related to the rotation of the displacement vector $u_{k}$, which results in vanishing of one material constant in equation (22). Some analogies with the theory of the so-called Cosserat pseudocontinuum [7] can be observed where the rotation (spin) vector of a body is not independently introduced but it is related to the rotation of the displacement vector.

Nevertheless, in the considered body no momentum stresses occur. The appearance of a non-symmetric part of the piezoelectric stress tensor is related to the presence of an electromagnetic field and to the non-vanishing of the antisymmetric part of the Maxwell stress tensor of that field as is indicated by (20) and (21).

The approach presented here enables the description of an isotropic piezoelectric based on the algebraic constitutive relations. In the symmetric theories of a deformable body in an electromagnetic field developed by Nowacki [6] and Mindlin [5] no piezoelectric effect in isotropic bodies with algebraic constitutive relations is obtained. Thus, it is necessary to introduce in the constitutive relations the gradients of polarization, as in the paper of Mindlin [5], and this increases the order of differential equations and complicates the problem.

A specific initial-boundary value problem together with an attempt to explain the Mead anomalies with the help of the method here presented will be treated elsewhere.

\section{References}

[1] J. D. Jackson, Classical Electrodynamics, Wiley, New York 1975.

[2] M. Kopisz, Coupled fields generated by linear differential operators: electrodynamics of deformable continua, Zastos. Mat. 20 (1988), 125-135.

[3] -, Principle of conservation of momentum, angular momentum and energy for a deformable continuum in an electromagnetic field, this volume, 349-359.

[4] C. A. Mead, Electron transport mechanism in thin insulting films, Phys. Rev. 128 (1962), 2088-2097. 
[5] R. D. Mindlin, Elasticity, piezoelectricity and crystal lattice dynamics, J. Elasticity 2 (1972), 217-236.

[6] W. Nowacki, Electromagnetic Phenomena in Deformable Solids, PWN, Warszawa 1983 (in Polish).

[7] -, Theory of Nonsymmetric Elasticity, PAN, Ossolineum 1970 (in Polish).

[8] W. Voight, Lehrbuch der Kristallphysik, Teubner, Leipzig 1910.

MAEGORZATA KOPISZ

INSTITUTE OF MATHEMATICS

TECHNICAL UNIVERSITY OF WROCEAW

WYBRZEŻE WYSPIAŃSKIEGO 27

50-370 WROCEAW, POLAND

Received on 15.5 .1990 